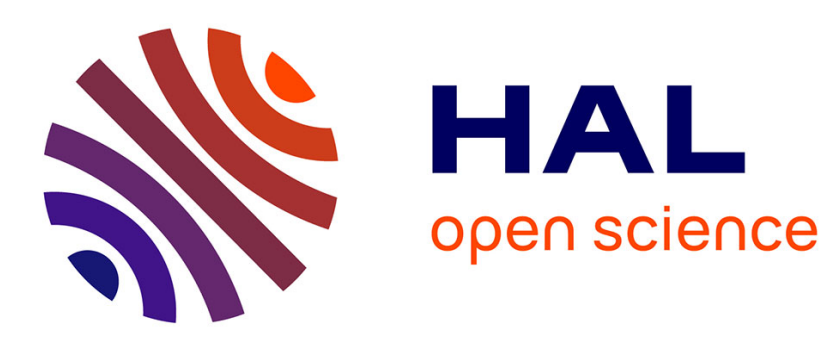

\title{
Evidence for exchange enhancement of TiBe2 from ESR
}

\author{
D. Shaltiel, P. Monod, I. Felner
}

\section{To cite this version:}

D. Shaltiel, P. Monod, I. Felner. Evidence for exchange enhancement of TiBe2 from ESR. Journal de Physique Lettres, 1980, 41 (23), pp.567-570. 10.1051/jphyslet:019800041023056700 . jpa-00231848

\section{HAL Id: jpa-00231848 https://hal.science/jpa-00231848}

Submitted on 1 Jan 1980

HAL is a multi-disciplinary open access archive for the deposit and dissemination of scientific research documents, whether they are published or not. The documents may come from teaching and research institutions in France or abroad, or from public or private research centers.
L'archive ouverte pluridisciplinaire HAL, est destinée au dépôt et à la diffusion de documents scientifiques de niveau recherche, publiés ou non, émanant des établissements d'enseignement et de recherche français ou étrangers, des laboratoires publics ou privés. 


\title{
Evidence for exchange enhancement of $\mathrm{TiBe}_{2}$ from $\mathbf{E S R}$
}

\author{
D. Shaltiel $(*)$, P. Monod and I. Felner ( $\left.{ }^{+}\right)$ \\ Laboratoire de Physique des Solides, Université Paris-Sud, 91405 Orsay, France \\ $(\dagger)$ Racah Institute of Physics, Hebrew University, Jerusalem, Israel. \\ (Reçu le 11 septembre 1980, accepté le 13 octobre 1980)
}

\begin{abstract}
Résumé. - Nous avons observé des signaux RPE très intenses dans $\mathrm{TiBe}_{2}$ de $1,4 \mathrm{~K}$ jusqu'à $300 \mathrm{~K}$, à $10 \mathrm{GHz}$. Les signaux présentent les caractéristiques suivantes : 1) forme de raie métallique ; 2) $g=1,98 \pm 0,01$ dans presque toute la gamme de températures; 3) la largeur de raie reste presque constante à 50 gauss, jusqu'à $10 \mathrm{~K}$. Puis elle augmente à peu près linéairement avec la température jusqu'à $150 \mathrm{~K}$, au taux de 3,7 gauss/K et augmente moins vite à plus haute température. Sur la base de ces observations, nous proposerons que $\mathrm{TiBe}_{2}$ est un métal à susceptibilité augmentée, plus grande que dans $\mathrm{Pd}$, en accord avec les mesures de susceptibilité, les mesures d'aimantation en haut champ, la chaleur spécifique et les résultats de diffraction de neutrons.
\end{abstract}

\begin{abstract}
We have observed very strong signals in $\mathrm{TiBe}_{2}$ from $1.4 \mathrm{~K}$ up to $300 \mathrm{~K}$ at $10 \mathrm{GHz}$. The signals have the following features : 1) metallic line shape ; 2) $g=1.98 \pm 0.01$ in most of the temperature range ; 3 ) the line width remains almost constant at $50 \mathrm{G}$ up to $10 \mathrm{~K}$, it then increases nearly linearly with temperature up to $150 \mathrm{~K}$ at a rate of $3.7 \mathrm{G} / \mathrm{K}$ and more slowly at higher temperatures. On the basis of this ensemble of observations we propose that $\mathrm{TiBe}_{2}$ is an enhanced susceptibility metal, like $\mathrm{Pd}$, in agreement with susceptibility, high field magnetization measurements, specific heat, and neutron diffraction results.
\end{abstract}

Unusual high susceptibilities in $\mathrm{TiBe}_{2}$ were first reported by Saji et al. [1]. It has recently attracted considerable attention due to the extensive investigation done by Matthias and his collaborators [2, 3, 4].

$\mathrm{TiBe}_{2}$ has a strong temperature dependent susceptibility [1] with a shallow maximum at about $10 \mathrm{~K}$ [2]. The $1 / \chi$ vs. temperature at low temperatures has a Curie-Weiss behaviour [5] with a slope corresponding to $1.6 \mathrm{Bohr}$ magnetons per formula unit and an intersection with the temperature axis at $-20 \mathrm{~K}$ [2]. These properties led Matthias et al. [2] to the conclusion that $\mathrm{TiBe}_{2}$ is an "itinerant antiferromagnet». Specific heat measurements show [3] that $\mathrm{TiBe}_{2}$ has a large density of states $\left(C / T=42 \mathrm{emu} / \mathrm{mole} . \mathrm{K}^{2}\right)$ and a small peak in the specific heat at $1.9 \mathrm{~K}$. If this peak is attributed to magnetic ordering it should correspond to about 0.02 Bohr magnetons per formula unit. Neutron diffraction results [4] at $1.6 \mathrm{~K}$ did not show any ordering of either a localized or non localized magnetic moment. From the neutron detection limit it was concluded that if any localization occurs it must be smaller than $0.2 \mu_{\mathrm{B}}$ per formula unit. Due to the lack of evidence for magnetic ordering

$\left({ }^{*}\right)$ Permanent address : Racah Institute of Physics, Hebrew University, Jerusalem, Israel. in the specific heat and neutron diffraction experiments and the indications that $\mathrm{TiBe}_{2}$ has a high density of states we have performed conduction electron spin resonance (CESR) experiments to obtain a better understanding of this compound. We found unexpectedly strong resonance signals from helium to room temperature. Analysis of the CESR results and the fact that they did not show any transition down to $1.4 \mathrm{~K}$ lead us to the conclusion that $\mathrm{TiBe}_{2}$ is a high susceptibility metal. Similar conclusions were reached from very recent magnetization measurements of $\mathrm{TiBe}_{2}$ at temperatures from $1.8 \mathrm{~K}$ to $10 \mathrm{~K}$ and at fields up to 19 tesla, where the susceptibility reveals a non linear behaviour [6].

The $\mathrm{TiBe}_{2}$ compound was prepared in an induction furnace using $99.9 \% \mathrm{Ti}$ and $\mathrm{Be}$. The sample was melted several times to obtain an homogeneous compound. It was not annealed after melting. The CESR was measured with a Varian spectrometer in a rectangular TE102 cavity from 4.2 to $300 \mathrm{~K}$ using a helium gas flow temperature controlled cryostat (Oxford Instruments). The accuracy of the temperature depended on the amount of helium flow, (the temperature is measured with a thermocouple close to but not in direct contact with the sample), it is about $\pm 1 \mathrm{~K}$ at low temperatures and between 
$\pm(2-4) \mathrm{K}$ above $50 \mathrm{~K}$. Additional measurements were done from 1.4 to $50 \mathrm{~K}$ in a home made $\mathrm{X}$ band spectrometer where the sample, a carbon resistor and a platinum resistor thermometer were in direct contact with the bottom of rectangular cavity. The temperatures were measured with an accuracy better than $\pm 0.1 \mathrm{~K}$ and $\pm 0.2 \mathrm{~K}$ below and above $10 \mathrm{~K}$ respectively.

The resonance lines of metallic shape (in the diffusionless limit i.e. $A / B \sim 2.5 \pm 0.6$ ) could be fitted very closely to a Lorentzian line shape with larger deviations in the high temperature region $T>200 \mathrm{~K}$. The line width, $\Delta H=1 / \gamma T_{2}$, extracted from the line shape is almost constant and about 50 gauss from 1.4 to $10 \mathrm{~K}$. Above $10 \mathrm{~K}$ the line width increases almost linearly with temperature with a slope of 3.7 gauss/K but it rises less rapidly above $140 \mathrm{~K}$. The line width at $300 \mathrm{~K}$ is 880 gauss (Fig. 1). The

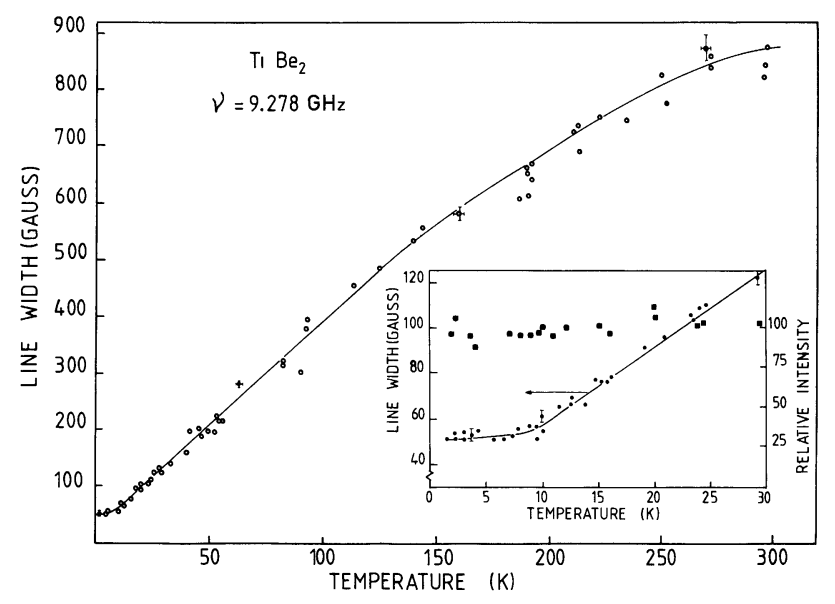

Fig. 1. - The CESR line width of $\mathrm{TiBe}_{2}$ as a function of temperature at $9.28 \mathrm{GHz}$. The insert gives a blow up of the low temperature region where the overall intensities $(\simeq 15 \%)$ are also shown (घ).

position of the line could be determined within $3 \%$ of the line width at low temperature and less accurately at temperatures above $200 \mathrm{~K}$. The $g$ value was $1.983 \pm 0.003$ at helium temperatures and

$$
1.985 \pm 0.005
$$

at $150 \mathrm{~K}$. A $g$ of $2.00 \pm 0.02$ was obtained at room temperature (Fig. 2). However due to the deviation from Lorentzian shape and the large errors we cannot deduce that there is a real increase in the $g$ value.

The experimental results do not show any transition that can be attributed to an antiferromagnetic ordering. The position of the line between 1.4 to $15 \mathrm{~K}$ is constant within the experimental error of \pm 3.5 gauss. The intensity of the resonance lines (measured with an accuracy of $\pm 15 \%$ ) was also practically constant below $15 \mathrm{~K}$ (Fig. 1). For an antiferromagnetic order-

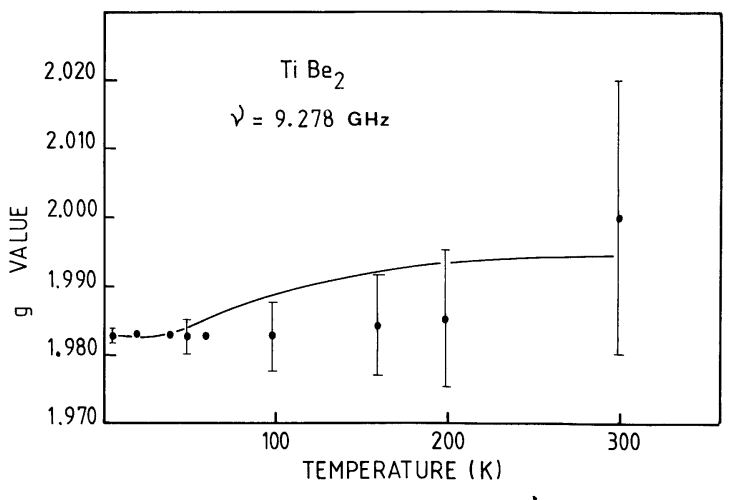

Fig. 2. - The CESR $g$ value of $\mathrm{TiBe}_{2}$ as a function of temperature at $9.28 \mathrm{GHz}$. Note the increase of the error with temperature due to the increase in the line width. Above $200 \mathrm{~K}$ deviations from a Lorentzian line shape increase the error even further. The full line gives the anticipated $g$ values obtained from equation (3).

ing one expects a shift of the resonance line of the order of $\left(2 H_{\mathrm{e}} H_{\mathrm{A}}\right)^{1 / 2}$ where $H_{\mathrm{e}}$ and $H_{\mathrm{A}}$ are the exchange fields and the anisotropy field respectively. An antiferromagnetic transition of $1.9 \mathrm{~K}$ as was suggested from the small peak in the specific heat [2] corresponds to $H_{\mathrm{A}} \approx 15000 \mathrm{G}$. A maximum shift of $3.5 \mathrm{G}$, derived from the limits of the experimental errors, gives an anisotropy field of $0.02 \mathrm{G}$ which is even smaller than the contribution due to dipolar anisotropy fields. Therefore it is very unlikely that $\mathrm{TiBe}_{2}$ is an antiferromagnet. An alternative possibility is that $\mathrm{TiBe}_{2}$ is an enhanced susceptibility metal. This would be in agreement with the high density of states obtained from specific heat, and the large temperature dependence of the susceptibility with its maximum at about $8 \mathrm{~K}$, similar to the high susceptibility metals such as $\mathrm{Pd}$ and $\mathrm{YCo}_{2}$. The non linear behaviour of the susceptibility at high fields observed recently [6] is also in agreement with the enhanced susceptibility picture.

Within this paramagnetic picture it is possible to analyse our data along the same lines as has already been followed for Pd [7], $\mathrm{ZrZn}_{2}$ [8] and $\mathrm{ScIn}_{3}$ [9]. At first we give an approximate determination of the exchange enhancement factor :

$$
\frac{1}{1+B_{0}}=\frac{1}{1-\lambda \chi_{0}} \cong 1+\lambda \chi
$$

by writing :

$$
\left[\frac{C}{T \chi_{0}}\right]_{\text {free electron }}=\left[\frac{C}{T \chi}\right]_{\mathrm{TiBe}_{2}}(1+\lambda \chi)
$$

where $\lambda$ is the exchange constant and $\chi_{0}$ and $\chi$ are the free electron and enhanced susceptibilities respectively. We can indeed determine this factor at $4.2 \mathrm{~K}$, using the measured values for $\mathrm{TiBe}_{2}$ of

$$
C / T=42 \mathrm{~mJ} / \mathrm{mol} . \mathrm{K}^{2} \quad[4]
$$


and $\chi=8.6 \times 10^{-3} \mathrm{emu} / \mathrm{mol}$. [2] compared to the free electron value :

$$
\left[\frac{C}{T \chi_{0}}\right]_{\text {free electrons }}=\frac{\pi^{2}}{3} k_{\mathrm{B}}^{2} / \mu_{\mathrm{B}}^{2}=7.29 \times 10^{8} \mathrm{deg}^{-2} \cdot \mathrm{G}^{-2} .
$$

We find $(1+\lambda \chi)\left(\mathrm{TiBe}_{2}\right)=15$ (underestimate).

Indeed this determination inherently assumes that the exchange enhancement only plays a role in the susceptibility, not in the specific heat. As this is only an approximation we can infer that this number is an underestimate of the exchange enhancement. (By comparison the same analysis for $\mathrm{Pd}$ would yield an enhancement factor of 5.0 instead of the generally accepted value of 10 .) From this, one can safely take the exchange enhancement of $\mathrm{Ti}-\mathrm{Be}_{2}$ to be at least in the range of 20 to 30 . We note however that this determination is only possible at the lowest temperatures where the specific heat can be safely extrapolated.

In the following we will therefore assume that the observed resonance lines are due to the conduction electron spin resonance CESR. The dominant contribution to the CESR line width arises from the spin flip of the electrons due to conduction-electron scattering in the presence of spin-orbit coupling.

The ratio of the rates of momentum change $\tau^{-1}$ and spin flip $T_{2}^{-1}\left(T_{2}=T_{1}\right)$ is given by the Elliot relation [10]

$$
T_{2}=\tau / \Delta g^{2}
$$

where $\Delta g$ is the $g$ shift with respect to the free electron $g$ value. Following Yafet [11] and Beuneu and Monod [7] we obtain from $\Delta H=1 / \gamma T_{2}$ and $\rho=m^{*} / n e^{2} \tau$ and equation (1) the relation :

$$
\Delta H(T)=\frac{n e^{2}}{\gamma m^{*}} \frac{\tau(T)}{T_{2}(T)} \rho(T)=\frac{n e^{2}}{\gamma m^{*}} \Delta g^{2} \rho(T) .
$$

Here $\Delta g$ is assumed to be temperature independent. The resistivity $\rho(T)$ of a sample obtained from the same batch as the ESR sample was measured from $1.4 \mathrm{~K}$ to $300 \mathrm{~K}$ (Fig. 3). A plot of $\Delta H(T)$ versus the

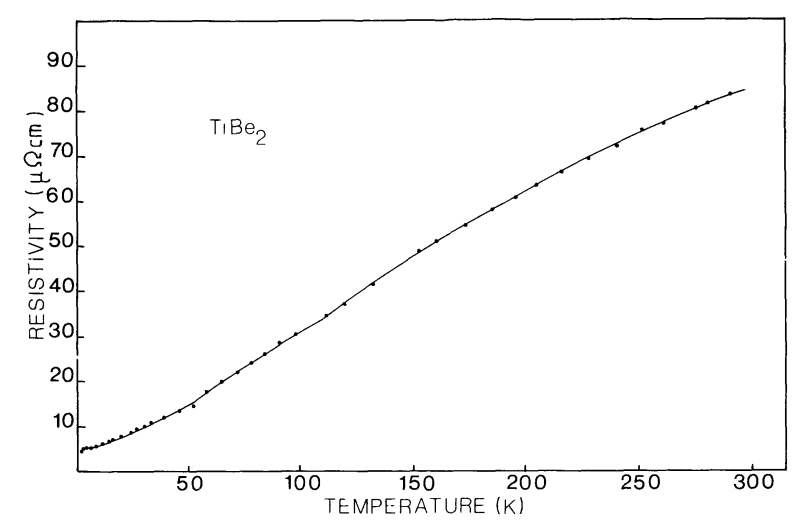

Fig. 3. - The resistivity of $\mathrm{TiBe}_{2}$ sample taken from the same batch as the samples on which the CESR was measured. resistivity $\rho(T)$ where $T$ is the implicit parameter shows an approximate linear relation between these two variables in the temperature range studied (Fig. 4). Although this result is in agreement with equation (2) we would like to point the following difficulties.

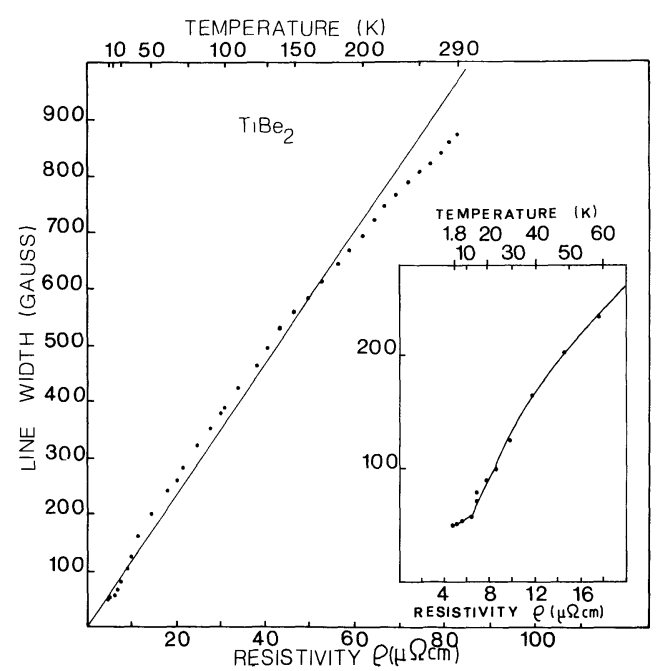

Fig. 4. - The line width as a function of the resistivity where the temperature $T$ was the implicit parameter. Note the approximate linear behaviour. The insert is a blow up of the low temperature region.

1) The deviation from linearity is of the order of $10 \%$ (Fig. 3).

2) The relatively narrow lines actually observed : Brinkman and Engelsberg [12] and Fulde and Luther [15] have shown that the CESR line in an exchange enhanced metal at low temperatures is narrowed by the exchanged enhanced constant $\frac{1}{1+B_{0}}=1+\lambda \chi$. We therefore deduce from the measured line width of $50 \mathrm{G}$ below $10 \mathrm{~K}$ that the line width without narrowing at helium temperature is about $1500 \mathrm{G}$. Walsh et al. [8] have extended the above results to higher temperature by proposing a life time enhancement factor of the form $T_{2}^{\prime}(T)=T_{2}(1+\lambda \chi(T))$. It is derived on the assumption that the relaxation is towards the instantaneous magnetization and it agrees with Brinkman and Engelsberg [11] results at low temperatures. They have used this model to explain their line width result in the itinerant ferromagnet $\mathrm{ZrZn}_{2}$ in its paramagnetic state, but in a very limited region $30 \mathrm{~K}$ to $60 \mathrm{~K}$. The same procedure in the itinerant ferromagnet $\mathrm{ScIn}_{3}$ has failed to give a quantitative agreement [9]. In both cases the temperature dependent contribution to the relaxation due to phonons in the presence of the spin-orbit coupling was not included. Adding to equation (2) the exchange narrowing proposed by Walsh [8], we obtain :

$$
\Delta H(T)=\frac{n e^{2}}{\gamma m^{*}} \frac{\rho(T)}{1+\lambda \chi(T)} \Delta g^{2} .
$$


A plot of $\Delta H(T)$ versus $\frac{\rho(T)}{1+\lambda \chi(T)}$ is shown in figure 5 . A linear relation is obtained in the very limited region $T<40 \mathrm{~K}$. At higher temperatures the extrapolated line width, assuming the linear behaviour derived from the low temperature region, is much larger than the experimental line width. The extrapolated line width at $300 \mathrm{~K}$ is about $5000 \mathrm{G}$ compared to the experimental value of $880 \mathrm{G}$. An assumption that $\Delta g$ decreases, with increasing temperature and hence $\Delta H$ should also decrease is out of our measured experimental error of the $g$ value in most of the temperature range (Fig. 2).

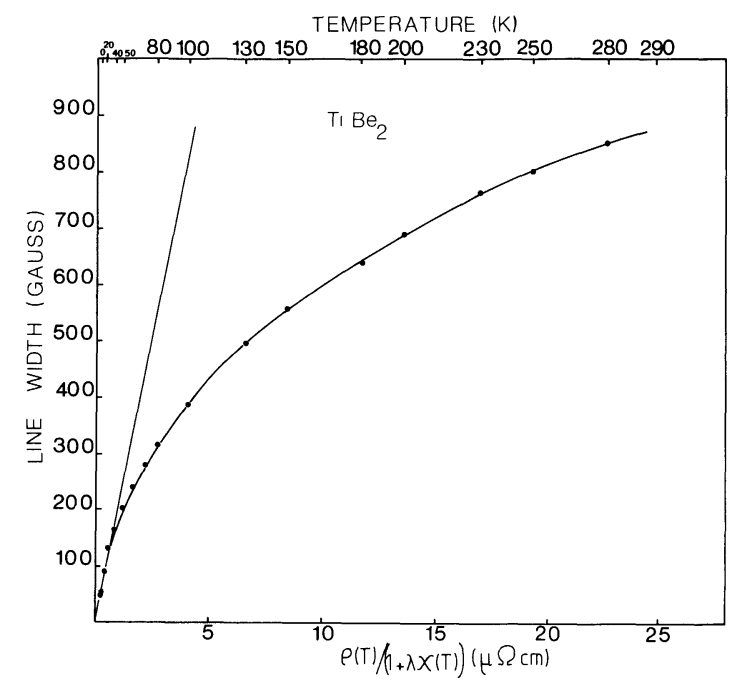

Fig. 5. - The line width as a function of the resistivity divided by $(1+\lambda \chi(T))$. A behaviour close to linear is observed in the low temperature region only, $T<40 \mathrm{~K}$. The straight line indicates the line width behaviour if the linearity had persisted at higher temperatures.

We have not so far an explanation for the behaviour of the line width in the whole temperature region. It is clear that Walsh et al. formula [8] for the life time enhancement factor does not hold (except may be for the low temperature region) in agreement also with the results in $\mathrm{ScIn}_{3}$ [9], mentioned earlier, that failed to show a quantitative agreement. Beuneu and Monod [7] have recently investigated the Elliot relation by plotting the known experimental data of
$\Delta H / \rho$ as a function of $\Delta g^{2}$ for all the metal elements where the CESR has been published. They obtained that most results fall within the boundaries given by $\Delta g^{2}=c \frac{\tau}{T_{1}}$ where $c$ varies between 0.05 and 1 . $\mathrm{Pd}$ is the exception where $c=10$. If the exchange narrowing enhancement is also included we obtain $c_{\mathrm{Pd}}($ exchanged narrowed $)=1$. Similarly for $\mathrm{TiBe}_{2}$ we obtain in the low temperature region using the results of figure 5, where the exchange narrowing is included $c_{\mathrm{TiBe}_{2}}$ (exchanged narrowed) $=1$. If we assume that it is very improbable that the linear relation $\Delta H / \rho v s . \Delta g^{2}$ for the large temperature region $T>130$ observed in figure 4 is accidental and therefore the Elliot relation is obeyed we obtain from equation (2) using an estimated enhancement for the electron mass of $1.5 \Delta g \approx 30 \frac{\tau}{T}$ e.g. $c \approx 30$. Hence if the boundary limits of $c$ mentioned earlier are valid an exchange narrowing of 30 , which is equal to the low temperature value, should be present in the high temperature region.

Finally we would like to mention the investigation of the CESR of $\mathrm{Ti}\left(\mathrm{Be}_{1-x} \mathrm{Cu}_{x}\right)_{2}$ for $x=0.01,0.05,0.1$ and 0.2. These compounds are ferromagnetic for $x \gtrsim 0.05[13,6]$. The resonance was observed in the ferromagnetic and paramagnetic states and will be reported elsewhere [15].

In conclusion the CESR results indicate that $\mathrm{TiBe}_{2}$ is an enhanced susceptibility metal. The CESR can be observed in a very large temperature range from $0 \mathrm{~K}$ to above room temperature and therefore it is possible to study the contributions of various interactions to the CESR properties of an enhanced susceptibility metal. And indeed our results indicate a complex behaviour. Though we show the existence of a relation between the CESR line width and the resistivity, this relation is not simple to interpret and further detailed study of the $\mathrm{TiBe}_{2}$ compound is necessary to obtain a better understanding of the system.

Acknowledgments. - We wish to thank H. Hurdequint for many helpful discussions and for his comments.

\section{References}

[1] Saji, H., Yamadaya, T., Asanuma, M., J. Phys. Soc. Japan 21 (1966) 255.

[2] Matthias, B. T., Giorgi, A. L., Struebing, V. O. and Smith, J. L., J. Physique Lett. 39 (1978) L-441.

[3] Sil.wart, G. R., Matthias, B. T., Giorgi, A. L., S/hI IR/, E. G. and Smith, J. L., Solid State Commun. 30 (1979) 109.

[4] Rackecha, V. C., Felcher, G. P., Sinha, S. K., Smith, J. L. and Matthias, B. T., Solid State Commun. 33 (1980) 495.

[5] The $1 / \chi$ vs. $T$ behaviour has an upwards bend at high temperatures (see reference [1]).

[6] Monod, P., Felner, I., Chouteau, G. and Shaltiel, D., J. Physique Lett. 41 (1980) L-511.
[7] Beuneu, F., Monod, P., Phys. Rev. B 18 (1978) 2422.

[8] Walsh Jr., W. M., Knapp, G. S., Rupp Jr., L. W. and SChmidt, P. H., J. Appl. Phys. 41 (1970) 1081.

[9] Dunifer, G. L., KNapp, G. S. and Corenzwitt, E., J. Appl. Phys. 41 (1970) 1075.

[10] Elliot, R. S., Phys. Rev. 96 (1954) 266.

[11] YAFET, Y., Solid State Phys. 14 (1963) 1.

[12] Brinkman, W. F. and Engelsberg, S., Phys. Rev. Lett. 16 (1968) 1187.

[13] Fulde, P. and Luther, A., Phys. Rev. 175 (1968) 337.

[14] Giorgi, A. L., Matthias, B. T., Stewart, G. R., A( hi R, F. and Smith, J. L., Solid State Commun. 32 (1979) 455.

[15] Monod, P., Shaltiel, D., in preparation. 